\title{
A New Method for Research on the Center-Focus Problem of Differential Systems
}

\author{
Zhengxin Zhou \\ School of Mathematical Sciences, Yangzhou University, Yangzhou 225002, China \\ Correspondence should be addressed to Zhengxin Zhou; zxzhou@yzu.edu.cn
}

Received 13 December 2013; Accepted 17 February 2014; Published 20 March 2014

Academic Editor: Douglas R. Anderson

Copyright (C) 2014 Zhengxin Zhou. This is an open access article distributed under the Creative Commons Attribution License, which permits unrestricted use, distribution, and reproduction in any medium, provided the original work is properly cited.

We will introduce Mironenko's method to discuss the Poincaré center-focus problem, and compare the methods of Lyapunov and Mironenko. We apply the Mironenko method to discuss the qualitative behavior of solutions of some planar polynomial differential systems and derive the sufficient conditions for a critical point to be a center.

\section{Introduction}

As we know $[1,2]$, for polynomial differential system

$$
\begin{aligned}
& \frac{d x}{d t}=-y+\sum_{i+j=2}^{n} p_{i j} x^{i} y^{j}, \\
& \frac{d y}{d t}=x+\sum_{i+j=2}^{n} q_{i j} x^{i} y^{j},
\end{aligned}
$$

where $p_{i j}$ and $q_{i j}$ are real constants, there has been a longstanding problem, called the Poincaré center-focus problem; for the system (1) find explicit conditions of $p_{i j}$ and $q_{i j}$ under which $(1)$ has a center at the origin $(0,0)$; that is, all the orbits nearby are closed. The problem is equivalent to an analogue for a corresponding periodic equation

$$
\frac{d r}{d \theta}=\frac{\sum_{i=0}^{n-2} A_{i}(\theta) r^{i}}{1+\sum_{i=0}^{n-1} B_{i}(\theta) r^{i}} r^{2}=R(\theta, r)
$$

to have periodic solutions. To see this let us note that the phase curves of $(1)$ near the origin $(0,0)$ in polar coordinates $x=r \cos \theta, y=r \sin \theta$ are determined by (2), where $A_{i}(\theta)$ and $B_{i}(\theta)$ are polynomials in $\cos \theta$ and $\sin \theta$.

Since the closed orbits of (1) correspond to $2 \pi$-periodic solutions of (2), the planar vector field (1) has a center at $(0,0)$ if and only if (2) has a center at $r=0$; that is, all the solutions nearby $r=0$ are periodic: $r(0)=r(2 \pi)[1,2]$.
To discuss the center-focus problem, there are Lyapunov's method and the others; see the works of Z. Zhang and so forth [2-8]. One of the most commonly used methods is Lyapunov's method. However, sometimes, the use of this method to calculate the focus quantities is very difficult and the amount of calculation is very large.

In this paper, we apply the method of Mironenko [9] (reflecting function method) to study the qualitative behavior of solutions of (2) and give the sufficient conditions for $r=$ 0 to be a center. We give a few examples to illustrate that, sometimes, Mironenko's method is better than Lyapunov's method.

In the present section, we introduce the concept of the reflecting function, which will be used throughout the rest of this paper.

Consider differential system

$$
x^{\prime}=X(t, x), \quad t \in R, x \in R^{n},
$$

which has a continuously differentiable right-hand side and with a general solution $\phi\left(t ; t_{0}, x_{0}\right)$. For each such system, the reflecting function is defined (see Mironenko's [9]) as $F(t, x):=\phi(-t, t, x)$. Therefore, for any solution $x(t)$ of $(3)$, we have $F(t, x(t))=x(-t), F(0, x)=x$.

If system (3) is $2 \omega$-periodic with respect to $t$, and $F(t, x)$ is its reflecting function, then $T(x):=F(-\omega, x)=\phi(\omega ;-\omega, x)$ is the Poincare mapping of (3) over the period $[-\omega, \omega]$. Thus, the solution $x=\phi\left(t ;-\omega, x_{0}\right)$ of (3) defined on $[-\omega, \omega]$ is $2 \omega$ periodic if and only if $x_{0}$ is a fixed point of $T(x)$. The stability 
of this periodic solution is equivalent to the stability of the fixed point $x_{0}$.

A differentiable function $F(t, x)$ is a reflecting function of system (3) if and only if it is a solution of the Cauchy problem

$$
F_{t}^{\prime}+F_{x}^{\prime} X(t, x)+X(-t, F)=0, \quad F(0, x)=x .
$$

If $F(t, x)$ is a reflecting function of system (3), then it is also reflecting function of the following systems:

$$
x^{\prime}=X(t, x)+\left(F_{x}^{\prime}\right)^{-1} G(t, x)-G(-t, F(t, x)),
$$

where $G(t, x)$ is an arbitrary vector function. Thus, the shift operators $[9,10]$ on interval $[-\omega, \omega]$ for such systems coincide, and the qualitative behaviors of the periodic solutions of these systems are the same. See papers [11-18] in which reflecting functions are also applied to study the qualitative behavior of solutions of some nonlinear and nonautonomous differential systems.

In the following, we always assume that all equations in this paper have a continuously differentiable right-hand side and have a unique solution for their initial value problem.

\section{Main Results}

Now, let us consider differential equation (2).

Lemma 1 ([see [9]). Suppose that $F(\theta, r)$ is the reflecting function of (2) and $F(\theta+2 \pi, r)=F(\theta, r)$. Then all the solutions of (2) defined on $[-\pi, \pi]$ are $2 \pi$-periodic; that is, $r=0$ is a center.

Lemma 2 ([see [9]). If all the solutions of (2) are $2 \pi$-periodic, then its reflecting function is $2 \pi$-periodic, too.

First, we introduce the Mironenko's method. Suppose that

$$
F=r+\sum_{i=1}^{\infty} a_{i}(\theta) r^{i}
$$

is the reflecting function of $(2)$, where $a_{i}(0)=0(i=1,2, \ldots)$. Substituting it into

$$
\frac{\partial F}{\partial \theta}+\frac{\partial F}{\partial r} R(\theta, r)+R(-\theta, F(\theta, r))=0
$$

and equating the coefficients of like powers of $r$, we get the following infinite equations

$$
\begin{gathered}
a_{1}^{\prime}(\theta)+A_{0}(\theta)+A_{0}(-\theta)=0 \\
a_{2}^{\prime}(\theta)+2 a_{1}(\theta)\left(A_{0}(\theta)+A_{0}(-\theta)\right)+A_{1}(\theta) \\
+A_{1}(-\theta)-A_{0}(\theta) B_{1}(\theta) \\
-A_{0}(-\theta) B_{1}(-\theta)=0 ;
\end{gathered}
$$

If $a_{i}(\theta+2 \pi)=a_{i}(\theta)(i=1,2, \ldots)$, then $F(\theta+2 \pi, r)=F(\theta, r)$ and $r=0$ is a center. If this is a $a_{k}(\theta+2 \pi) \neq a_{k}(\theta)$ and $a_{i}(\theta+$ $2 \pi)=a_{i}(\theta)(i=1,2, \ldots, k-1)$, then $a_{k}(\theta)=\varphi_{k}(\theta)+h_{k}$, where $\varphi_{k}(\theta+2 \pi)=\varphi_{k}(\theta), h_{k} \neq 0$ is a constant and when $h_{k}<0$, $(0,0)$ is an unstable focus; when $h_{k}>0,(0,0)$ is a stable focus.
By the above description, it seems that Mironenko's method is similar to Lyapunov's method, but do not forget that reflecting function has some good symmetry properties, which make our calculations greatly reduced. The following example will illustrate this advantage.

Example 3. Consider system

$$
\begin{aligned}
& \dot{x}=-2 y+x^{2}-x y-2 y^{2}-x y^{2}-y^{3}, \\
& \dot{y}=2 x+3 x y-y^{2}+x y^{2}-y^{3} .
\end{aligned}
$$

Taking $x=r \cos \theta, y=r \sin \theta$, it is transformed to equation

$$
\frac{d r}{d \theta}=\frac{\cos \theta-\sin \theta(1+r \sin \theta)}{1+(1+r \sin \theta)^{2}} r^{2} .
$$

Now, we use the method of Lyapunov to discuss is the $r=$ 0 a center or focus? Suppose that

$$
r=c+\sum_{i=1}^{\infty} b_{i}(\theta) c^{i}, \quad b_{i}(0)=0, \quad i=1,2, \ldots
$$

is a solution of (10). Substituting it into (10), we get

$$
\begin{gathered}
b_{1}^{\prime}(\theta)+b_{2}^{\prime}(\theta) c+b_{3}^{\prime}(\theta) c^{2}+\cdots+b_{n}^{\prime}(\theta) c^{n-1}+\cdots \\
=\frac{1}{2}(\cos \theta-\sin \theta) \psi^{2}-\frac{c}{2} \sin \theta \cos \theta \psi^{3} \\
+\frac{c^{2}}{4} \sin ^{2} \theta(\cos \theta+\sin \theta) \psi^{4} \\
-\frac{c^{3}}{4} \sin ^{4} \theta \psi^{5}-\frac{c^{4}}{8} \sin ^{4} \theta(\cos \theta+\sin \theta) \psi^{6} \\
+\frac{c^{5}}{8} \cos \theta \sin ^{5} \theta \psi^{7}+\cdots
\end{gathered}
$$

where $\psi:=1+b_{1}(\theta) c+b_{2}(\theta) c^{2}+\cdots+b_{n}(\theta) c^{n}+\cdots$.

Equating the coefficients of like powers of $c$ implies

$$
b_{1}^{\prime}(\theta)=\frac{1}{2}(\cos \theta-\sin \theta) .
$$

Solving it we get

$$
\begin{aligned}
& b_{1}(\theta)=\frac{1}{2}(\sin \theta+\cos \theta-1), \\
& b_{2}^{\prime}(\theta)=\frac{1}{2}(\cos 2 \theta-\cos \theta+\sin \theta-\sin \theta \cos \theta) .
\end{aligned}
$$


It implies

$$
\begin{aligned}
& b_{2}(\theta)= \frac{1}{2}\left(\sin \theta \cos \theta-\sin \theta-\cos \theta+1-\frac{1}{2} \sin ^{2} \theta\right), \\
& b_{3}^{\prime}(\theta)= \frac{1}{2} \sin ^{3} \theta+\frac{3}{4}\left(\cos \theta-\cos ^{2} \theta-\sin \theta\right. \\
&\left.\quad-2 \sin ^{2} \theta \cos \theta+\sin ^{2} \theta+\sin \theta \cos \theta\right), \\
& b_{3}(\theta)= \frac{1}{4}\left(3 \sin \theta+\cos \theta-3 \sin \theta \cos \theta-2 \sin ^{3} \theta\right. \\
& b_{4}^{\prime}(\theta)=\left.+\frac{3}{2} \sin ^{2} \theta+\frac{2}{3} \cos ^{3} \theta-\frac{5}{3}\right) \\
&+\frac{5}{8} \cos \theta \sin ^{3} \theta+\frac{11}{4} \sin ^{2} \theta \cos \theta+\frac{3}{8} \sin ^{4} \theta \\
&+\frac{1}{6} \cos { }^{4} \theta-\frac{2}{3} \cos \theta+\frac{2}{3} \sin \theta-\frac{13}{8} \sin ^{2} \theta \cos ^{2} \theta \\
&+\frac{1}{12} \sin \theta \cos ^{2} \theta \\
& \sin \cos ^{3} \theta-\frac{1}{4} \cos ^{3} \theta-\frac{3}{4} \sin ^{3} \theta
\end{aligned}
$$

so

$$
\begin{aligned}
b_{4}= & -\frac{1}{2} \sin ^{2} \theta-\frac{1}{3} \cos ^{3} \theta+\frac{5}{32} \sin ^{4} \theta \\
& +\sin ^{3} \theta-\frac{11}{12} \sin \theta-\frac{1}{48} \cos ^{4} \theta+\frac{1}{12} \cos \theta \\
& +\frac{13}{48} \sin \theta \cos 2 \theta+\frac{31}{48} \sin \theta \cos \theta+\frac{13}{48} .
\end{aligned}
$$

From above, we see that the calculation of the expression of $b_{i}(\theta)(i=5,6,7, \ldots)$ will become more difficult. Thus, it is not easy to judge is $r=0$ a center?

Now, we try to use the method of Mironenko to discuss is $r=0$ a center? In (7) taking

$$
R=\frac{\cos \theta-\sin \theta(1+r \sin \theta)}{1+(1+r \sin \theta)^{2}} r^{2},
$$

we get

$$
\begin{aligned}
& a_{1}^{\prime}(\theta)+a_{2}^{\prime}(\theta) r+\cdots+a_{n}^{\prime}(\theta) r^{n-1}+\cdots \\
& +\left(1+2 a_{1}(\theta) r+3 a_{2}(\theta) r^{2}+\cdots+(n+1) a_{n}(\theta) r^{n}+\cdots\right) \\
& \times\left(\frac{1}{2}(\cos \theta-\sin \theta)-\frac{r}{2} \sin \theta \cos \theta\right. \\
& +\frac{r^{2}}{4} \sin ^{2} \theta(\cos \theta+\sin \theta)-\frac{r^{3}}{4} \sin ^{4} \theta \\
& \left.\quad-\frac{r^{4}}{8} \sin ^{4} \theta(\cos \theta-\sin \theta)+\frac{r^{5}}{8} \cos \theta \sin ^{4} \theta+\cdots\right) \\
& +\frac{1}{2}(\cos \theta+\sin \theta) \varphi^{2}+\frac{r}{2} \cos \theta \sin \theta \varphi^{3}
\end{aligned}
$$

$$
\begin{aligned}
& +\frac{r^{2}}{4} \sin ^{2} \theta(\cos \theta-\sin \theta) \varphi^{4} \\
& -\frac{r^{3}}{4} \sin ^{4} \theta \varphi^{5}-\frac{r^{4}}{8} \sin ^{4} \theta(\cos \theta+\sin \theta) \varphi^{6} \\
& -\frac{r^{5}}{8} \cos \theta \sin ^{5} \theta \varphi^{7}+\cdots=0 .
\end{aligned}
$$

Equating the coefficients of like powers of $r$ implies

$$
\begin{array}{ll}
a_{1}^{\prime}(\theta)=-\cos \theta, & a_{1}(\theta)=-\sin \theta \\
a_{2}^{\prime}(\theta)=2 \sin \theta \cos \theta, & a_{2}(\theta)=\sin ^{2} \theta \\
a_{3}^{\prime}(\theta)=-3 \sin ^{2} \theta \cos \theta, & a_{3}(\theta)=-\sin ^{3} \theta \\
a_{4}^{\prime}(\theta)=4 \sin ^{3} \theta \cos \theta, & a_{4}(\theta)=\sin ^{4} \theta
\end{array}
$$

and so forth. Now we can conjecture that $a_{i}(\theta)=$ $(-\sin \theta)^{i}, i=1,2,3, \ldots$; that is, $F(\theta, r)=r /(1+r \sin \theta)$. It is not difficult to check that $F(\theta, r)=r /(1+r \sin \theta)$ is a solution of (7), so it is the reflecting function of (10). As $F(\theta+2 \pi, r)=F(\theta, r)$, so the origin point $(0,0)$ of system $(9)$ is a center.

Such example shows us that, sometimes, the method of Mironenko is better than Lyapunov's method. Just this advantage is not enough. By the equivalence, we know that $F(\theta, r)=r /(1+r \sin \theta)$ also is the reflecting function of the following equations:

$$
\begin{aligned}
\frac{d r}{d \theta}= & \frac{\cos \theta}{1+(1+r \sin \theta)^{2}} r^{2}+(1+r \sin \theta)^{2} G(\theta, r) \\
& -G\left(-\theta, \frac{r}{1+r \sin \theta}\right),
\end{aligned}
$$

where $G(\theta, r)$ is an arbitrary function. If we take

$$
G(\theta, r)=\frac{r^{2 k-1}}{(1+r \sin \theta)\left(1+(1+r \sin \theta)^{2}\right)^{k}},
$$

where $k$ is a positive integer, then (20) becomes

$$
\frac{d r}{d \theta}=\frac{r^{2} \cos \theta\left(1+(1+r \sin \theta)^{2}\right)^{k-1}+r^{2 k-1}\left(1+r \sin \theta-r^{2}\right)}{\left(1+(1+r \sin \theta)^{2}\right)^{k}} .
$$

Taking $x=r \cos \theta, y=r \sin \theta$, then (22) is transformed to

$$
\begin{aligned}
\frac{d x}{d t}= & \left(x^{2}-2 y-2 y^{2}-y^{3}\right)\left(2+2 y+y^{2}\right)^{k-1} \\
& +x\left(x^{2}+y^{2}\right)^{k-1}\left(1+y-x^{2}-y^{2}\right), \\
\frac{d y}{d t}= & x\left(2+3 y+y^{2}\right)\left(2+2 y+y^{2}\right)^{k-1} \\
& +y\left(x^{2}+y^{2}\right)^{k-1}\left(1+y-x^{2}-y^{2}\right)
\end{aligned}
$$

and its origin point $(0,0)$ is a center too.

Thus, by using the method of Mironenko we not only solve a center-focus problem, but at the same time open a 
class of differential equations with the same character of point $r=0$. Therefore, we can say, sometimes, the method of Mironenko is more effective than Lyapunov's method.

Theorem 4. If $F(\theta, r)$ is a solution of equation $R(\theta, r)=$ $R(-\theta, F)$ such that $F(0, r)=r$ and satisfies (7), then all the solutions of (2) determined on $[-\pi, \pi]$ are $2 \pi$-periodic; that is, $r=0$ is a center.

Proof. By the assumptions, we see that $F(\theta, r)$ is a reflecting function of (2) and $2 \pi$-periodic; thus by Lemma 1 , the present conclusion is correct.

Theorem 5. If there is a function $(\theta, r)(\partial u / \partial r \neq 0), u(\theta+$ $2 \pi, r)=u(\theta, r)$ which satisfies

$$
\frac{\partial u}{\partial \theta}+\frac{\partial u}{\partial r} R(\theta, r)=f(\theta, u)
$$

where $f(\theta, u)+f(-\theta, u)=0$. Then all the solutions of (2) determined on $[-\pi, \pi]$ are $2 \pi$-periodic; that is, $r=0$ is a center.

Proof. Let $F(\theta, r)$ be the reflecting function of (2). Now, we prove that it satisfies $u(\theta, r)=u(-\theta, F)$. have

In fact, by relation (24), for any solution $r(\theta)$ of $(2)$, we

$$
\frac{d u(\theta, r(\theta))}{d \theta}=f(\theta, u(\theta, r(\theta)) .
$$

In view of $f(\theta, u)+f(-\theta, u)=0$, we have [9, page 65]

$$
u(\theta, r(\theta))=u(-\theta, r(-\theta))
$$

that is,

$$
u(\theta, r)=u(-\theta, F)
$$

which implies that $F(\theta, r)$ is a $2 \pi$-periodic function with respect to $\theta$. By Lemma 1 , the proof of the present theorem is finished.

Corollary 6. If there is a differentiable $2 \pi$-periodic function $a(\theta)$ such that

$$
\begin{aligned}
& \alpha_{0}:=a^{\prime}(\theta)+\sum_{j=0}^{n} b_{j}(\theta)(-a(\theta))^{j}, \\
& \alpha_{k}:=\sum_{j=k}^{n} b_{j}(\theta) C_{j}^{k}(-a(\theta))^{k-j}, \quad(k=1,2, \ldots, n)
\end{aligned}
$$

$\left(C_{n}^{k}=n ! / k !(n-k) !\right)$ are $2 \pi$-periodic and odd functions, then all the solutions defined on $[-\pi, \pi]$ of equation

$$
\frac{d r}{d \theta}=\sum_{j=0}^{n} b_{j}(\theta) r^{j}:=Y(\theta, r)
$$

are $2 \pi$-periodic.

This result is implied by Theorem 5 if we take $u=a(\theta)+r$ and put

$$
\frac{\partial u}{\partial \theta}+\frac{\partial u}{\partial r} Y(\theta, r)=\sum_{j=0}^{n} \alpha_{j} u^{j}
$$

Example 7. Taking $a(\theta)=\sin \theta$, we get that all the solutions determined on $[-\pi, \pi]$ of the Riccati equation

$$
\begin{aligned}
\frac{d r}{d \theta}= & \sin ^{2} \theta \cos \theta-\cos \theta+\left(\sin \theta \cos \theta+2 \sin ^{4} \theta\right) r \\
& +\sin ^{3} \theta r^{2}
\end{aligned}
$$

are $2 \pi$-periodic.

Theorem 8. For (2), suppose that

$$
\begin{array}{r}
\sum_{i=0}^{k}\left(A_{i}(\theta) B_{k-i}(-\theta)+A_{i}(-\theta) B_{k-i}(\theta)\right)=0 \\
k=1,2, \ldots, 2 n-3,
\end{array}
$$

$A_{i}(\theta)=0(i>n-2), B_{j}(\theta)=0(j>n-1), B_{0}(\theta)=1$. Then $r=0$ is a center.

Proof. By the assumptions, it is not difficult to check that $F(\theta, r)=r$ is the reflecting function of (2), so $r=0$ is a center.

Theorem 9. For (2), suppose that $\int_{0}^{\pi}\left(A_{0}+\bar{A}_{0}\right) d \theta=0$ and

$$
\begin{gathered}
\sum_{j=0}^{k}\left(B_{j}\left(A_{0}+\bar{A}_{0}\right)-A_{j}\right) \sum_{i=0}^{k-j} \bar{B}_{i} C_{n-1-i}^{k-j-i} \alpha^{k-j-i} \\
=\sum_{j=0}^{k}\left(B_{j}+B_{j-1} \alpha\right) \sum_{i=0}^{k-j} \bar{A}_{i} C_{n-2-i}^{k-j-i} \alpha^{k-j-i}, \\
k=1,2, \ldots, 2 n-2, \\
B_{0}=1, \quad A_{i}=0, \quad(i>n-2), \\
B_{j}=0, \quad(j>n-1),
\end{gathered}
$$

where $\alpha=\int_{0}^{\theta}\left(A_{0}+\bar{A}_{0}\right) d s, \bar{A}_{i}=A_{i}(-\theta), \bar{B}_{i}=B_{i}(-\theta)(i=$ $0,1,2, \ldots, 2 n-2)$. Then all the solutions of (2) and the following equations

$$
\frac{d r}{d \theta}=R(\theta, r)+(1+r \alpha)^{2} G(\theta, r)-G\left(-\theta, \frac{r}{1+r \alpha}\right)
$$

determined on $[-\pi, \pi]$ are $2 \pi$-periodic; that is, $r=0$ is a center, where $G(\theta, r)$ is a continuously differentiable $2 \pi$ periodic function with respect to $\theta$ and $G(\theta, 0)=G(-\theta, 0)$.

Proof. By condition (33), we can check that $F=r /(1+r \alpha)$ is the reflecting function of (2). As $\int_{0}^{\pi}\left(A_{0}+\bar{A}_{0}\right) d \theta=0$, so $\alpha$ and $F=r /(1+r \alpha)$ are $2 \pi$-periodic functions with respect to $\theta$. Then by Lemma $1, r=0$ is a center.

Theorem 10. Suppose that for (2) $r=0$ is a center and functions $\Delta_{k}(\theta, r)$ are $2 \pi$-periodic with respect to $\theta$ and satisfy

$$
\Delta_{\theta}+\Delta_{r} R(\theta, r)=R_{r}(\theta, r) \Delta(\theta, r), \quad \Delta(\theta, 0)=0 .
$$


Then for the following equations

$$
\frac{d r}{d \theta}=R(\theta, r)+\sum_{k=0}^{\infty} \alpha_{k}(\theta) \Delta_{k}(\theta, r),
$$

$r=0$ is a center, too. Here $\alpha_{k}(\theta)(k=0,1,2, \ldots)$ are arbitrary continuously differentiable $2 \pi$-periodic odd functions.

Proof. By the assumptions and $[9,14]$, we know that the reflecting functions of (2) and (36) coincide and all the solutions of (2) determined on $[-\pi, \pi]$ are $2 \pi$-periodic. By Lemma 2, we know that the common reflecting function is $2 \pi$-periodic; thus all the solutions of (36) determined on $[-\pi, \pi]$ are $2 \pi$-periodic; that is, $r=0$ is a center, too.

Remark 11. Theorem 10 shows us that if the center-focus problem for (2) is already solved, then the same problem for (36) is solved, too.

\section{Conflict of Interests}

The author declares that there is no conflict of interests regarding the publication of this paper.

\section{Acknowledgments}

The work supported by the NSF of Jiangsu of China under Grant no. BK2012682 and the NSF of China under Grant nos. $11271026,61374010$.

\section{References}

[1] M. A. M. Alwash and N. G. Lloyd, "Nonautonomous equations related to polynomial two-dimensional systems," Proceedings of the Royal Society of Edinburgh A, vol. 105, pp. 129-152, 1987.

[2] Z. Zhang, Qualitative Theory of Differential Equations, Beijing Science Press, Beijing, China, 1998.

[3] J. Li, "Hilbert's 16th problem and bifurcations of planar polynomial vector fields," International Journal of Bifurcation and Chaos in Applied Sciences and Engineering, vol. 13, no. 1, pp. 47106, 2003.

[4] A. P. Sadowskii, Polynomial Ideals and Varieties, University Press, Minsk, Belarus, 2008.

[5] I. A. Cherkas, "Center conditions of a equation," Differentsial'nye Uravneniya, vol. 13, no. 2, pp. 271-275, 1977.

[6] C. Li, C. Liu, and J. Yang, "A cubic system with thirteen limit cycles," Differentsial'nye Uravneniya, vol. 246, no. 9, pp. 36093619, 2009.

[7] Y.Zhao, Z. Liang, and G. Lu, "The cyclicity of the period annulus of the quadratic Hamiltonian systems with non-Morsean point," Differentsial'nye Uravneniya, vol. 162, no. 1, pp. 199-223, 2000.

[8] C. Qian, "Asymptotic behavior of a third-order nonlinear differential equation," Journal of Mathematical Analysis and Applications, vol. 284, no. 1, pp. 191-205, 2003.

[9] V. I. Mironenko, Analysis of Reflective Function and Multivariate Differential System, University Press, Gomel, Belarus, 2004.

[10] V. I. Arnold, Ordinary Differential Equation, Science Press, Moscow, Russia, 1971.

[11] V. I. Mironenko, "A reflecting function of a family of functions," Differentsial'nye Uravneniya, vol. 36, no. 12, pp. 1636-1641, 2000.
[12] L. A. Alisevich, "On linear system with triangular reflective function," Differentsial'nye Uravneniya, vol. 25, no. 3, pp. 14461449, 1989.

[13] E. V. Musafirov, "Differential systems, the mapping over period for which is represented by a product of three exponential matrixes," Journal of Mathematical Analysis and Applications, vol. 329, no. 1, pp. 647-654, 2007.

[14] V. V. Mironenko, "Time-symmetry-preserving perturbations of differential systems," Differentsial'nye Uravneniya, vol. 40, no. 10, pp. 1325-1332, 2004.

[15] S. V. Maiorovskaya, "Quadratic systems with a linear reflecting function," Differentsial'nye Uravneniya, vol. 45, no. 2, pp. 263$265,2009$.

[16] Z. Zhou, "On the reflective function of polynomial differential system," Journal of Mathematical Analysis and Applications, vol. 278, no. 1, pp. 18-26, 2003.

[17] Z. Zhou, "The structure of reflective function of polynomial differential systems," Nonlinear Analysis. Theory, Methods \& Applications A, vol. 71, no. 1-2, pp. 391-398, 2009.

[18] Z.Zhou, "Research on the properties of some planar polynomial differential equations," Applied Mathematics and Computation, vol. 218, no. 9, pp. 5671-5681, 2012. 


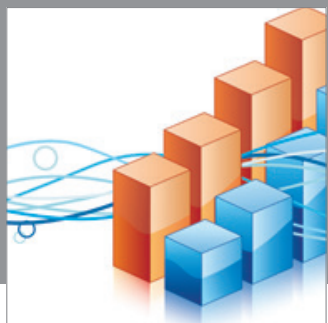

Advances in

Operations Research

mansans

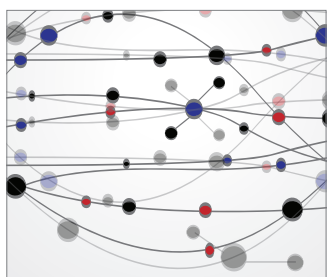

The Scientific World Journal
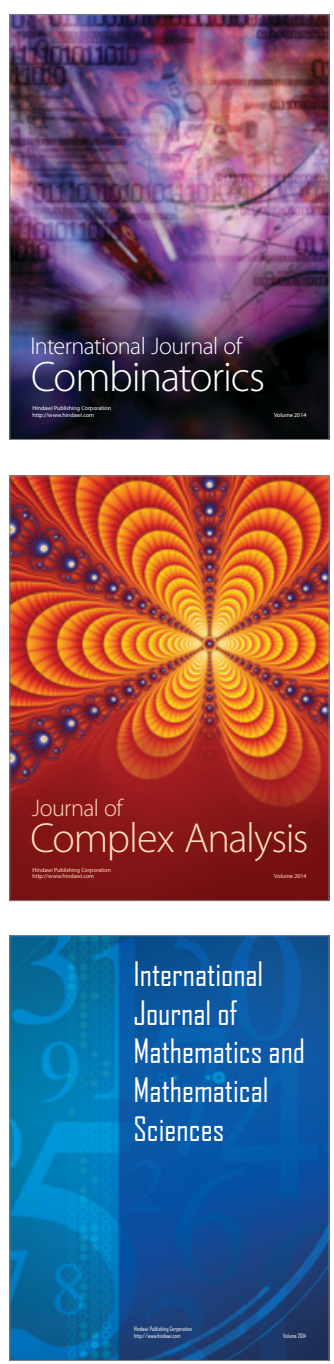
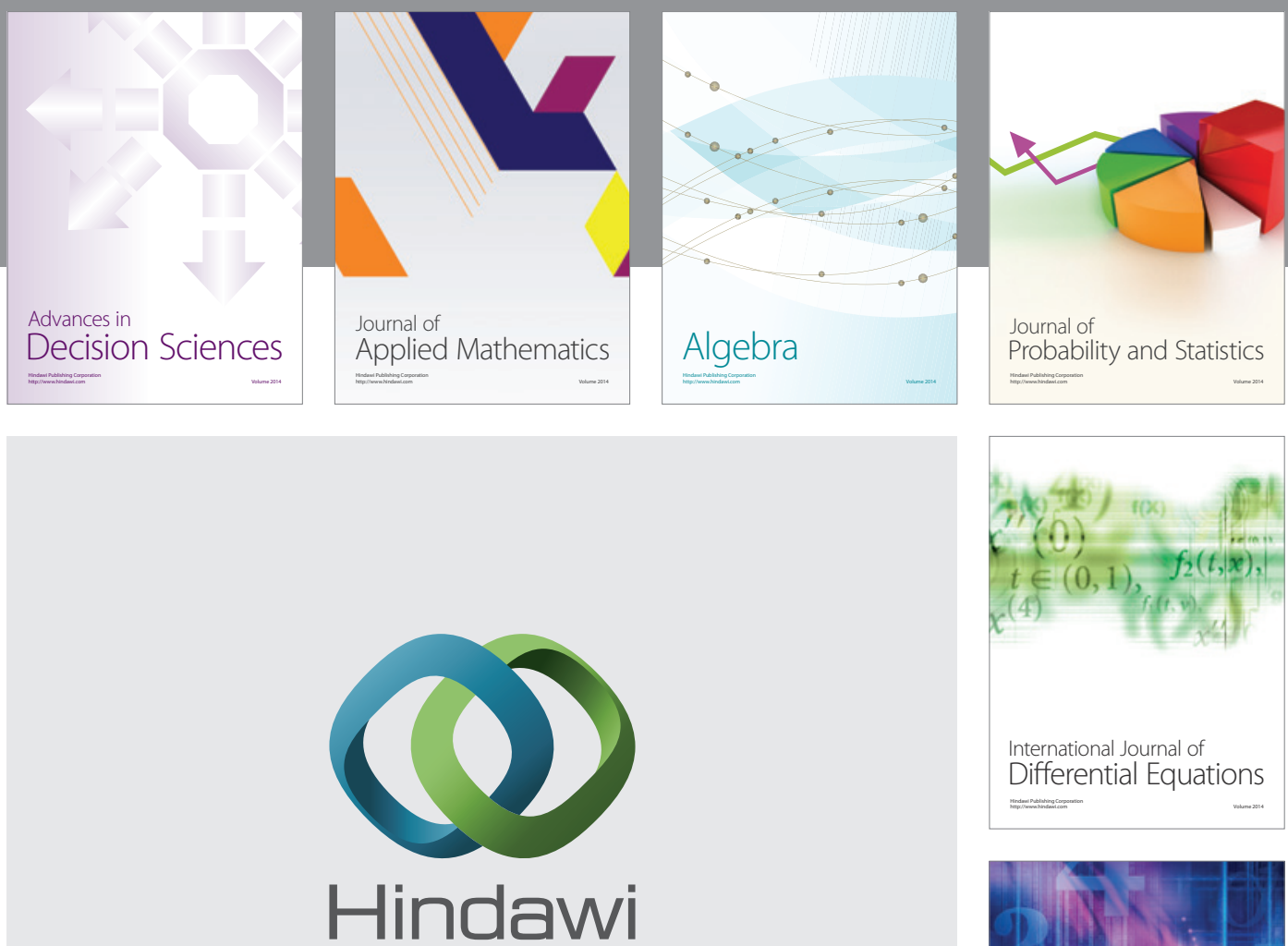

Submit your manuscripts at http://www.hindawi.com
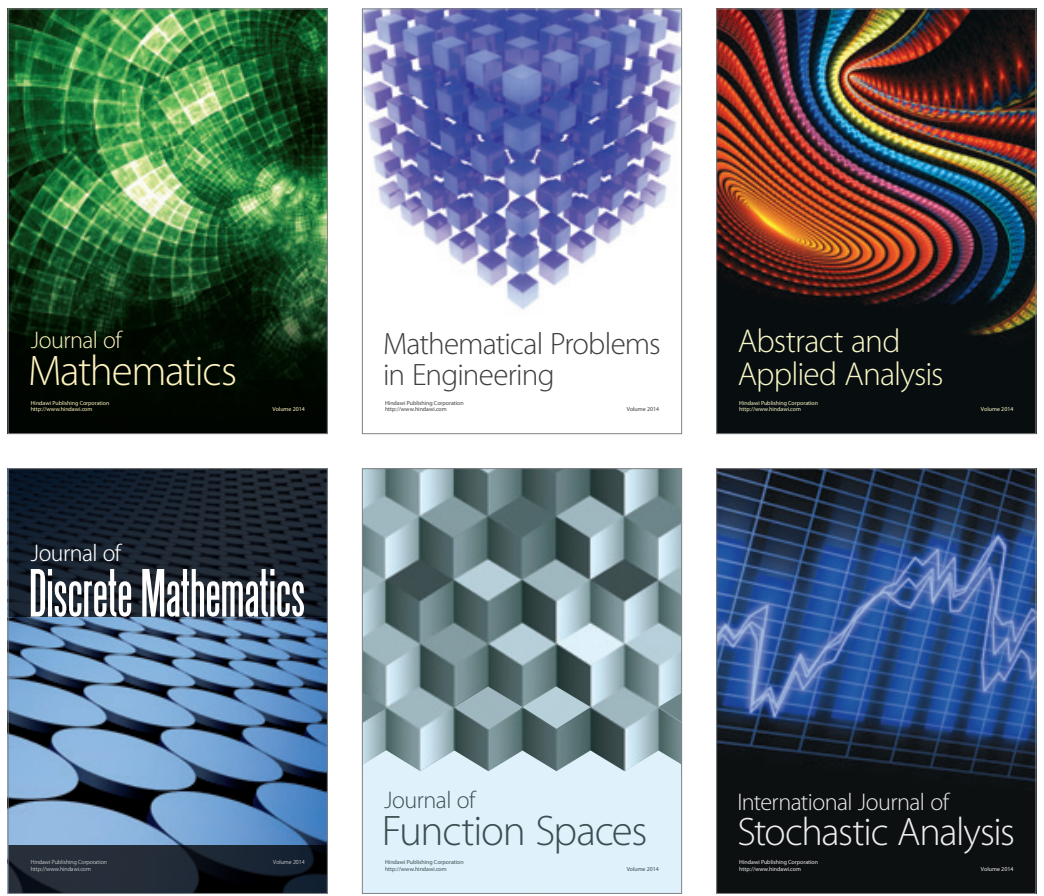

Journal of

Function Spaces

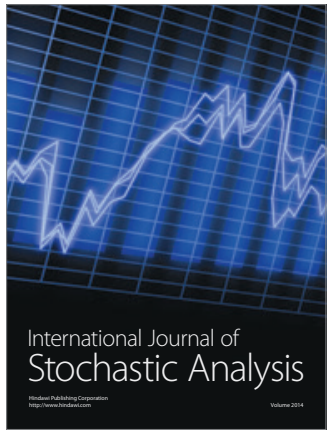

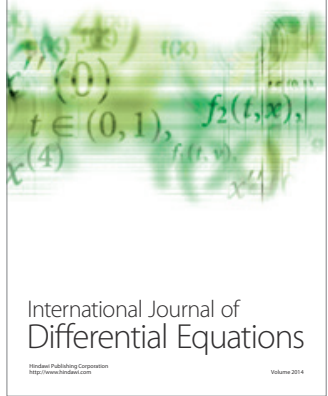
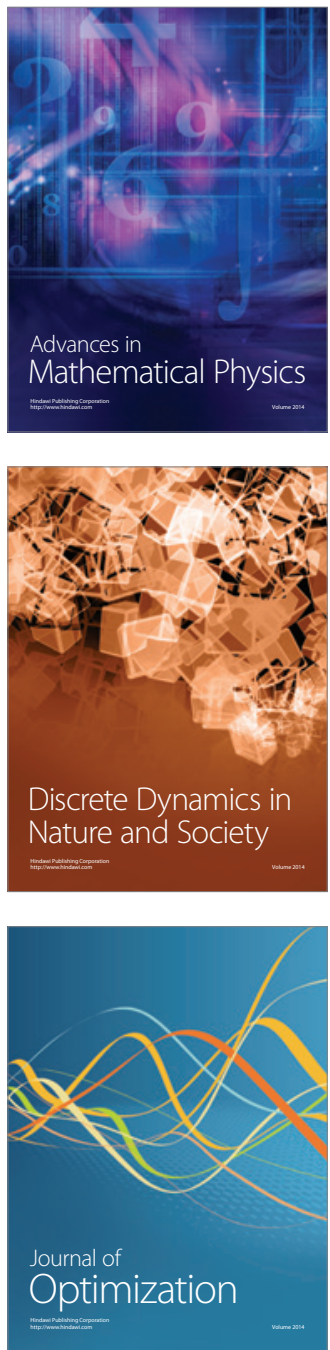